\title{
Procesamiento semántico de palabras epistémicas y metafísicas en niños y adolescentes con Trastorno de Espectro Autista (TEA) y con Desarrollo Típico (DT)
}

\section{Semantic processing of epistemic and metaphysical words in children and adolescents with Autism Spectrum Disorder (ASD) and Typically Developing (TD)}

\author{
Karen L. Juillerat \\ Felipe A. Cornejo \\ Ramón D. Castillo \\ Laboratorio de Psicología experimental, Facultad de Psicología, Universidad de Talca, Chile \\ Sergio E. Chaigneau \\ Centro de Investigación de la Cognición (CINCO), Facultad de Psicología, Universidad Adolfo Ibáñez, Santiago, Chile
}

(Rec: 19 junio 2013 / Acept: 27 octubre 2015)

\begin{abstract}
Resumen
La capacidad para entender palabras abstractas se relaciona con la inteligencia y procesos de orden superior. Sin embargo, un creciente número de investigaciones sugiere que las palabras abstractas, aun cuando tienen bajos niveles de concreción e imaginabilidad, son procesadas de una manera diferente. Este artículo proporciona evidencia experimental, donde el procesamiento de palabras abstractas-epistémicas (v.g. imaginación, certeza) es diferente al procesamiento de palabras abstractas-metafísicas (v.g. libertad, criterio). Se llevó a cabo un experimento en el que 16 niños y adolescentes con Trastorno del Espectro Autista (ASD) y un grupo pareado con Desarrollo Típico (DT) completaron nueve sentencias incompletas graduadas por dificultad. Los sujetos con TEA fueron menos precisos y lentos con palabras epistémicas-abstractas, y lentos con palabras abstractas-metafísicas que los sujetos con DT. Los resultados se discuten en términos de los procesos cognitivos y sociales para detectar y entender los estados mentales, una habilidad llamada teoría de la mente (ToM).

Palabras clave: razonamiento, procesamiento semántico y teoría de la mente.
\end{abstract}

\begin{abstract}
The ability to understand abstract words is related to intelligence and higher order processes. However, a growing corpus of research suggests that abstracts words, while having lower level of concreteness and imaginability, are processed in different manners. This article provides experimental evidence that the processing of epistemic-abstract words (eg. Imagination, certainty) is different from the processing of metaphysicalabstract words (eg. Freedom, criteria). We carried out an experiment in which 16 children and adolescents with Autism Spectrum Disorder (ASD) and a typically developing matched group (TD) completed nine sentences graded by difficulty. Subjects with ASD were less accurate and slower with abstract-epistemic words; and slower with abstract-metaphysical words, than subjects with TD. The findings are discussed in terms of the social and cognitive processes to detect and understand the mental states, an ability named theory of mind (ToM).

Keywords: reasoning, semantic processing, theory of mind.
\end{abstract}

\footnotetext{
Agradecimientos: Parte de los experimentos y mediciones de esta investigación se realizaron con el patrocinio del Proyecto Fondecyt 우 1070155. Correspondencia: debe ser dirigida a: Ramón D. Castillo, Facultad de Psicología, Universidad de Talca, Avenida Lircay s/n, Talca, Chile. Tel (5671) 2201567, Fax (5671) 2201510, email: racastillo@utalca.cl, http:\psicologia.utalca.cl
} 


\section{Introducción}

La disminuida habilidad para detectar estados mentales y razonar con teoría de la mente (ToM), son los mecanismos propuestos para explicar el déficit adaptativo que presentan las personas con autismo y síndrome de Asperger (BaronCohen, Leslie y Frith, 1985 y 1986). Sin embargo, este déficit no sólo afecta el razonamiento, sino que se extiende a otros dominios, tales como la memoria, el lenguaje y las habilidades de comprensión lectora (Brown, Oram-Cardy y Johnson, 2013; Loukusa et al., 2007; Toichi y Kamio, 2002 y 2003; Dennis, Lazenby y Lockyer, 2001). Los antecedentes teóricos recopilados y la evidencia de nuestros experimentos apuntan a demostrar que estos déficits tienen en común una incapacidad general para integrar claves sociales en la interpretación y desambiguación de los significados, lo cual afecta diferencialmente el procesamiento de palabras.

Desde que fue propuesto que los niños autistas carecían de una teoría de la mente, múltiples investigaciones clínicas y experimentales han estado orientadas a describir y explicar el desarrollo de los mecanismos cognitivos subyacentes a la adaptación social (Baron-Cohen, Leslie y Frith, 1985 y 1986; Happé, 1993, 1994 y 1995; Tirassa, Bosco y Colle, 2006). Dichas investigaciones han comparado personas de desarrollo típico (DT) con grupos clínicos, en especial personas diagnosticadas con trastornos del espectro autista (TEA) (Baron-Cohen et al., 1985; Siegel, Minshew y Goldstein, 1996). Las personas con TEA usualmente son caracterizadas como inhábiles para entender los estados mentales de los otros, traduciéndose en una serie de problemas de ajuste social y comunicación. Este déficit además, según el DSM-IV, sería concomitante con intereses y patrones de conductas estereotipadas y con la ausencia de juego creativo e imaginación (American Psychiatric Association, 1994).

Para que el razonamiento con ToM sea adaptativo requiere la activación secuencial de precursores atencionales, perceptuales y motores, tales como un mecanismo detector de intenciones, seguido por un detector de la dirección de las miradas y un mecanismo de atención conjunta (Araya, Araya, Chaigneau, Martínez y Castillo, 2009; Charman, Baron-Cohen, Swettenham, Baird, Cox y Drew, 2000; Lecannelier, 2004; Liebal, Behne, Carpenter y Tomasello, 2009). Este último es un precursor no sólo para la ToM, sino también para la adquisición del lenguaje. Compartir la atención con otros implica la comprensión tácita que las personas son agentes intencionales y para adquirir el lenguaje el niño debe interpretar las palabras y los gestos comunicativos de los otros como actos intencionales (Liebal et al., 2009).
Así, la capacidad de atención conjunta define la base para captar intencionalidad y la conciencia de sí mismo. Además establece una vía para que los niños puedan aprender sobre el entorno a través de la perspectiva de los otros (Tomasello, 1996). En la captación de intencionalidad son identificadas dos habilidades: una de ellas más rudimentaria, que aparece en los primeros meses, la cual permite comprender a otros como agentes intencionales (Onishi y Baillargeon, 2005) y otra más sofisticada que aparece alrededor de los 4 años de edad, que permite la comprensión de los otros como agentes con contenidos mentales (Tager-Flusberg, 1992; Sullivan, Zaitchik y Tager-Flusberg, 1994).

El razonamiento con ToMy el procesamiento semántico en niños con TEA.

La atención conjunta, la capacidad para detectar intenciones y la comprensión de los contenidos mentales están asociadas, a la habilidad global para entender los significados. Esta relación está fundamentada por la evidencia que muestra que los déficits en algunas de estas habilidades son concomitantes con un amplio rango de déficits en el lenguaje, incluyendo no sólo las habilidades pragmáticas, sino también las léxicas-semánticas (Tesink et al., 2011).

El procesamiento léxico-semántico consiste en otorgar significados a una determinada situación, acción, objeto o símbolo. Aun cuando las palabras tienen un correlato con determinadas experiencias motoras y sensoriales, la relación entre la palabra y su significado es arbitraria. De ahí entonces que la adquisición de palabras en general y las palabras que refieren estados mentales (epistémicas) requieren extraer el significado a partir de claves propioceptivas y en especial, integrar el contexto social (Papafragou, Cassidy y Gleitman, 2007; Symons, 2004). Los términos que aluden a estados mentales pueden dividirse en palabras que se refieren a los estados cognitivos, deseos y estados emocionales. Los estados cognitivos dan cuenta de creencias y otros acontecimientos mentales, e incluyen verbos tales como pensar, saber, recordar, olvidar y fingir, entre otros. Los términos de deseo incluyen verbos tales como querer, ansiar, esperar, desear y necesitar. Y por último, los términos que aluden a estados emocionales se vinculan con palabras como feliz, triste, enojado, dañado, entusiasmado y satisfecho (Symons, 2004).

En general, los niños con autismo proveen menos términos que aluden a estados mentales en el discurso espontáneo, comparados con niños normales y con síndrome de Down (Baron-Cohen et al., 1986; Tager-Flusberg, 1992). Alrededor de los 4 años de edad, los niños con desarrollo típico son capaces de reconocer verbos que aluden a estados mentales 
como pensar, saber, soñar, fingir, esperar, desear o imaginar. Estos verbos son fácilmente diferenciados de los verbos no mentales, como saltar, comer o mover, sin embargo los niños con autismo presentan mucha más dificultad en hacer esta diferenciación (Baron-Cohen et al., 1994).

Ziatas, Durkin y Pratt (1998) examinaron la relación de ToM con el desarrollo y uso de los enunciados de creencia comparando niños con autismo, niños con síndrome de Asperger (SA), niños con trastornos específicos del lenguaje (TEL) y niños con desarrollo típico (DT). Aplicaron la clásica tarea de la falsa creencia usada por Baron-Cohen y colaboradores (1985), en conjunto con la prueba de comprensión de enunciados de creencia desarrollada por Moore, Bryant y Furrow (1989). Esta última prueba requería que el niño identificara la posición de un objeto tomando en consideración las pistas dadas por dos títeres, los cuales usaban los enunciados de creencia pensar, saber y adivinar. Para responder correctamente era necesario que el niño comprendiera que la palabra saber posee más certeza que las palabras pensar o adivinar. Finalmente fue aplicada una tarea de expresión de enunciados de creencia, donde el niño fue instruido para que él mismo controlara los títeres, mientras usaba las 3 palabras mencionadas anteriormente con el fin de ayudar al experimentador a encontrar el objeto. En la primera tarea de falsa creencia, la mayoría de los niños autistas contestaron erróneamente, mientras que la mayor parte de niños con SA, TEL y DT aprobaron. En la tarea de comprensión de enunciados de creencias, los niños autistas nuevamente tuvieron un desempeño menor que sus pares de los otros grupos, los cuales no difirieron entre sí. Finalmente, en la tarea de expresión de enunciados de creencia, se confirmó que los niños con autismo tenían un desempeño menor que sus pares, lo cual indica que los niños con autismo presentan un deterioro más severo en su habilidad para identificar, entender y usar los estados mentales, que los niños con SA y el resto de sus pares.

Dennis et al. (1999) estudiaron a un grupo de niños con TEA de alto funcionamiento intelectual y niños con SA, dos grupos de niños con fenilcetonuria y un grupo de niños con daño cerebral a nivel frontal. Los grupos fueron evaluados con pruebas de inteligencia tales como diseño con bloques y una escala de comprensión. Se encontró que los niños con TEA y un subgrupo de niños con fenilcetonuria tuvieron un bajo nivel de comprensión, aun cuando su desempeño en la prueba de diseño con bloques fue normal.

En otro estudio, Dennis, Lazenby y Lockyer (2001) compararon un grupo formado por niños con autismo de alto funcionamiento intelectual y síndrome de Asperger con un grupo control múltiple de niños con desarrollo normal típico. Ambos grupos fueron similares en tareas no inferenciales que requerían manejo adecuado de vocabulario e interpretación de frases ambiguas. Sin embargo, los niños con TEA tuvieron un desempeño menor que el grupo control en tareas que requerían hacer inferencias pragmáticas basadas en reglas de implicación; en inferir coherentemente a partir de guiones sociales cotidianos; comprender metáforas y modismos sociales; y finalmente, una menor habilidad para producir actos del discurso. La importancia de estos hallazgos reside en que los sujetos con TEA fueron capaces de identificar intenciones, pero no pudieron captar su significado utilizando el contexto social. Del mismo modo, captar la ambigüedad semántica les fue más fácil que entender las metáforas. Indicando que el problema para las personas con TEA fue detectar la intencionalidad del enunciado, más que el hecho que ciertos conceptos tenian significados múltiples. Además las normas sociales fueron más fáciles de entender que los actos de habla, sugiriendo que la dificultad de los sujetos con TEA es comprender la intencionalidad en los contextos sociales, más que la coherencia lógica de normas sociales.

Estos déficits en la esfera del procesamiento léxico semántico también se extienden al contexto académico. Un estudio meta-analítico llevado a cabo por Brown, Oram-Cardy y Johnson (2013) reveló que la comprensión de lectura en niños con TEA aparece disminuida en comparación con un grupo igualado de niños con DT. Sin embargo, esta disminución tiene un rango amplio de variabilidad que puede ir desde una habilidad marcadamente deficitaria a una habilidad dentro de rangos normales, dependiendo de los contenidos semánticos involucrados. A partir de este meta-análisis se constató que las habilidades de decodificación y puntajes de inteligencia en el área manual fueron similares entre niños TEA y niños con DT, aun cuando los niños con TEA aparecían disminuidos en el conocimiento semántico. En otro aspecto en el cual convergieron los estudios analizados fue que independiente del tipo de niño (con TEA o con DT), solo el conocimiento semántico y la decodificación fueron buenos predictores de los puntajes de la comprensión lectora, dado que los puntajes de inteligencia en el área manual no lo fueron. Finalmente, hubo convergencia en señalar que los niños con TEA tenían más dificultades comprendiendo textos que demandan interpretar claves sociales que textos que no demandan la interpretación de dichas claves.

En términos del procesamiento semántico ha sido demostrado que los adolescentes y adultos con TEA tienen un patrón atípico, el cual no sólo les dificulta la comprensión de estados mentales, metáforas y aspectos socio-pragmáticos del lenguaje, sino que se extiende a tareas que involucran 
la memoria. Por ejemplo, las personas con DT recuerdan mejor las palabras codificadas semánticamente que las palabras codificadas perceptualmente, porque el procesamiento de contenido verbal facilita más el recuerdo de conceptos que el procesamiento de aspectos superficiales como los fonológicos o perceptuales. Empero, en las personas con TEA el recuerdo de las palabras procesadas de manera perceptual (gráfica o fonológica) es similar al recuerdo de las palabras procesadas semánticamente. Es decir, la jerarquía con predominio de lo semántico por sobre lo perceptual no está presente en personas con TEA (Toichi y Kamio, 2002). Algo similar ocurre con el procesamiento semántico y la memoria a largo plazo, específicamente en el recuerdo de palabras abstractas y concretas. Mientras las personas con desarrollo típico recuerdan mejor las palabras concretas que las abstractas, en el grupo de personas con TEA el recuerdo no muestra ningún predominio (Toichi y Kamio, 2003).

En una investigación llevada a cabo por Harris y colaboradores (2006), fue analizado el procesamiento de palabras abstractas y concretas en personas con TEA y un grupo cuidadosamente igualado de adultos con DT. Para seleccionar las palabras fueron tomados en cuenta los parámetros de significado, imaginabilidad y concreción implementados por Paivio, Yuille y Madigan (1968). Basado en estos parámetros, las palabras pueden ser jerarquizadas desde la más a la menos concreta. En un extremo están las palabras con un claro referente sensorial y fácilmente imaginables tales como insecto o metal, mientras que en el otro extremo están las palabras abstractas que difícilmente se pueden imaginar y para las cuales no existe un referente sensorial, tales como criterio o vacuidad. En su investigación, Harris y su grupo diferenciaron las palabras abstractas en palabras epistémicas que aludían a estados mentales (recuerda, percibe, sospecha, vergüenza y expectativa) y palabras metafísicas, las cuales describían entidades socialmente consensuadas que están fuera de la corporalidad de las personas y los objetos (justicia, libertad, seguridad, influencia y desafío). En dicho estudio fue analizado el procesamiento semántico y perceptual de las palabras. Para el procesamiento semántico, los sujetos debían evaluar la palabra en términos de su valencia (positiva y negativa). Para el procesamiento perceptual, en cambio, los sujetos debían responder si la palabra estaba escrita con letras mayúsculas o minúsculas. En términos de desempeño, no fueron detectadas diferencias entre los grupos (TEA versus DT) en la proporción de respuestas con valencia positiva y tampoco en los tiempos de reacción. Sólo fueron detectadas diferencias por tipos de palabras, donde las palabras epistémicas recibieron significativamente menos valoraciones positivas y marginalmente tuvieron menores tiempos de reacción que las palabras metafísicas y concretas. La activación cerebral para palabras epistémicas y metafísicas no fue diferente entre sí, motivo por el cual fueron agrupadas en un solo conglomerado de palabras abstractas. Haciendo esta distinción, sólo fueron detectadas diferencias entre los grupos en la activación cerebral para el procesamiento semántico y perceptual de palabras concretas y abstractas (Harris et al., 2006).

De los antecedentes expuestos, al menos tres ideas en las cuales hay consenso pueden ser esbozadas: (a) La inteligencia, a diferencia del razonamiento ToM, no está completamente asociada a la adaptación social. Como ha sido planteado hasta ahora, las personas con TEA de alto funcionamiento tienen habilidades intelectuales en un rango considerado como normal. Sin embargo, sus problemas de ajuste social se correlacionan con su desempeño deficitario en tareas que miden razonamiento con ToM. (b) Complementariamente, es posible afirmar que el razonamiento con ToM, aun cuando es crucial para la adaptación humana, sigue una trayectoria independiente a las habilidades intelectuales. Incluso personas con alteraciones genéticas y metabólicas, con un nivel intelectual deficitario, a veces son capaces de identificar y razonar con estados mentales, lo que facilita su adaptación social. (c) Finalmente, las personas con TEA comparadas con las personas con DT tienen una modalidad de procesamiento cualitativamente distinta, que se extiende en varios dominios cognitivos. Especialmente en tareas de reconocimiento semántico, memoria y comprensión que requieren interpretar claves sociales o haberlas incorporado para desambiguar los contenidos y significados.

A pesar de este relativo acuerdo, hay un vacío explicativo acerca de cómo el procesamiento léxico-semántico y la inteligencia por un lado y la interpretación de claves sociales y el razonamiento ToM por el otro, están conectados. Parte del vacío apunta a la falta de consistencia entre el desarrollo del lenguaje y la tipología para diferenciar palabras en abstractas y concretas. Las palabras en general se adquieren en la relación con otros significativos, a partir de la coordinación de la percepción, la actividad motora y la experiencia propioceptiva. En este contexto social de la adquisición del lenguaje, las palabras concretas se aprenden tempranamente y con más rapidez que las palabras abstractas, porque poseen un claro referente sensorial con el cual la percepción del objeto, la acción sobre éste y la propiocepción se pueden coordinar, referente sensorial que las palabras abstractas no poseen. Visto de esta manera, las palabras abstractas requerirían de más interacción con los otros para ser entendidas y usadas. Sin embargo, esa habilidad para interactuar con otros está disminuida en los niños con 
TEA, porque precursores como la atención conjunta operan disfuncionalmente. Así entonces, tomando en cuenta que las palabras abstractas tienen un componente social mayor y un menor componente sensorial que las palabras concretas. Es posible hipotetizar que las palabras abstractas en general y sin ninguna distinción serían adquiridas tardíamente y usadas con dificultad por los sujetos con TEA.

Sin embargo, hay varios elementos que permiten afirmar que las palabras abstractas no son un grupo homogéneo. Por ejemplo, cierta evidencia indica que existe un subgrupo de palabras abstractas que aluden a estados mentales (epistémicas), que serían procesadas de manera distinta al resto. Sabemos por ejemplo que las palabras epistémicas son entendidas y usadas tempranamente por niños con DT, mientras que son adquiridas tardíamente o usadas con dificultad por niños diagnosticados con TEA. También es reconocido que las personas con TEA de alto funcionamiento intelectual tienen un desempeño cualitativamente distinto en un amplio dominio de funciones cognitivas, en comparación con los sujetos con DT. Esta diferencia cualitativa tendría que ver fundamentalmente con el déficit en la interpretación de claves sociales, que no es compensado con los altos niveles de inteligencia verbal. Así, si es asumido que el grupo de palabras abstractas es heterogéneo, donde las palabras que aluden a estados mentales tienen una modalidad de procesamiento distinta del resto y no están del todo conectadas a la inteligencia, entonces deberíamos hipotetizar que los sujetos con TEA de alto nivel intelectual deberían ser capaces de entender y usar las palabras abstractas, con excepción de aquellas que aluden a estados mentales.

Tal como fue detallado anteriormente, esta hipótesis fue contrastada por Harris y colaboradores (2006) con resultados modestos, encontrando que en términos de desempeño conductual, independiente del grupo (TEA vs DT), las palabras abstractas-epistémicas tuvieron una menor proporción de valencias positivas y fueron procesadas levemente más rápido que las palabras abstractas-metafísicas y concretas. No obstante, a pesar de las tendencias observadas, tenemos reparos al paradigma experimental implementado por Harris y colaboradores. Básicamente los reparos apuntan a que los protocolos para el estudio del funcionamiento cerebral requieren que los estímulos sean fáciles de procesar, dado que los tiempos de exposición a técnicas de imágenes cerebrales deben ser reducidos. En este caso, pedirle a la persona una valencia (negativa o positiva) para una palabra o pedirle que clasifique perceptualmente las palabras en mayúsculas o minúsculas, es menos demandante que pedirle definir la palabra. Alguien podría desconocer el significado de una palabra y asignarle una valencia aleatoriamente. Del mismo modo, clasificar una palabra en términos del tipo de letra es algo que se puede hacer sin conocer su significado. Las palabras, a su vez, tienen distintos grados de dificultad, lo que podría estar dado, entre otros aspectos, por la frecuencia de uso o el texto (discurso) en el cual las palabras están insertas.

Proponemos que las diferencias conductuales entre sujetos con TEA y DT pueden ser acentuadas cuando se les pide conocer el significado de una palabra inserta en una oración y cuando estas palabras previamente han sido graduadas por dificultad. Para eso llevamos a cabo la construcción de un dispositivo experimental, en el que fueron graduados los niveles de dificultad de las palabras a partir de las respuestas que nos dieron los niños y adolescentes con DT. Este dispositivo, una vez ensamblado, fue aplicado a niños y adolescentes con TEA y con DT, quienes habían sido igualados en variables sociodemográficas y de funcionamiento intelectual.

En suma, el objetivo de esta investigación fue analizar el funcionamiento cognitivo de sujetos con TEA (con autismo o con síndrome de Asperger), de alto funcionamiento intelectual, ante el procesamiento de frases u oraciones que incluían palabras concretas, abstractas-epistémicas y abstractas-metafísicas graduadas por dificultad, bajo la hipótesis general que aunque sus habilidades intelectuales sean similares a sujetos con DT, deberían presentar un procesamiento deficiente de palabras que aluden a estados mentales, mientras que no deberían tener problemas para procesar las palabras que aluden a entidades abstractas metafísicas y menos aún en el procesamiento de palabras concretas.

\section{Fase 1: Diseño y calibración de estímulos experimentales}

\section{Método}

Dos objetivos guiaron esta fase. El primero de ellos fue probar el nivel de comprensión de las palabras clasificadas como concretas, abstractas-metafísicas y abstractasepistémicas, usando como referente las palabras utilizando en el estudio de Harris y colaboradores (2006). El segundo objetivo fue clasificar las palabras por nivel de dificultad, dependiendo del número de niños con DT que contestaron correctamente. Las palabras seleccionadas por medio de este procedimiento fueron la base para diseñar el experimento definitivo. Además se esperó encontrar evidencia respecto a que las palabras abstractas-epistémicas y abstractas-metafísicas 
fuesen más difíciles de procesar que las palabras concretas, tomando en cuenta que los parámetros de imaginabilidad y concreción de estas palabras son menores.

\section{Participantes}

Fueron seleccionados por disponibilidad 10 escolares de enseñanza básica de la ciudad de Talca, 3 mujeres y 7 hombres, cuyas edades fluctuaron entre los 12 y 14 años $(M=13.1, D E=0.73)$.

\section{Materiales e instrumentos}

La tarea experimental fue ejecutada y registrada en un computador portátil con una pantalla de video de 14 pulgadas. Para desplegar aleatoriamente las palabras experimentales se usó el programa E-prime (versión 1.2; Psychology Software Tools, Inc., Pittsburgh, PA). Este software permite diseñar, generar y reproducir experimentos computarizados; además de recolectar, editar y analizar datos, teniendo la precisión de milisegundos para asegurar la exactitud de los datos. El mismo programa fue utilizado en la segunda fase y en el experimento definitivo.

\section{Procedimiento}

En un computador portátil fueron proyectadas, con la asistencia de un investigador, 120 palabras que previamente habían sido clasificadas en tres categorías: concretas (40), abstractas-metafísicas (40) y abstractas-epistémicas (40). Las palabras fueron presentadas en orden aleatorio y el participante debía responder cuál era su significado, sinónimo o hacer una descripción de los eventos asociados a la palabra. Cada participante disponía de 10 segundos para responder, si contestaba antes de ese tiempo el investigador apretaba la barra espaciadora para continuar con la siguiente palabra. Una vez pasados 10 segundos, la palabra cambiaba automáticamente a la siguiente. Cada respuesta fue registrada por el investigador en una hoja-plantilla, clasificando si el participante presentaba o no, comprensión de la palabra. Si el sujeto entregaba un sinónimo, una descripción, una explicación o un ejemplo, su respuesta era clasificada con un uno (1). Las respuestas erradas, sobre-generalizaciones, o el reconocer no saber el significado era clasificado con un cero (0). Los participantes respondieron en una sala habilitada, sentados frente al computador. Las palabras estaban divididas en 2 etapas, en la primera se exponían al azar 60 palabras, en la cual las tres categorías estaban distribuidas proporcionalmente. Inmediatamente finalizada la primera parte se exponían las siguientes 60 palabras. El experimento duró entre 15 y 25 minutos por alumno. Para cada palabra fue contabilizado el número de sujetos que contestó correctamente. Para cada tipo de palabra (concretas, abstractas-metafísicas, abstractas-epistémicas) fue calculado el promedio de sujetos que contestó correctamente.

\section{Resultados y discusión}

El promedio de la proporción de escolares que contestó correctamente en cada tipo de palabra fue sometido a un análisis de varianza, detectándose diferencias significativas entre los tipos de palabras: $\mathrm{F}(2.27)=14.83 ; \mathrm{p}<.01$. Comparaciones múltiples con la prueba de diferencias mínimas significativas (DMS) mostraron que las palabras concretas tuvieron un promedio de aciertos mayor $(\mathrm{M}=$ $8.92, \mathrm{DE}=0.98)$ que las palabras abstractas-epistémicas $(\mathrm{M}=7.64, \mathrm{DE}=1.87)$ y abstractas-metafísicas $(\mathrm{M}=7.07$, $\mathrm{DE}=1.77)$, ps $<.01$. No fueron halladas diferencias entre los dos tipos de palabras abstractas: $\mathrm{p}>.40$.

Los sujetos tendieron a definir de mejor manera las palabras concretas que las abstractas. Además, al definir palabras abstractas, sean metafísicas o epistémicas, no hubo diferencias. Lo anterior corrobora la idea que las palabras concretas, dados sus niveles de imaginabilidad y concreción, son más fáciles de definir, explicar o ejemplificar que las palabras abstractas (Paivio et al., 1968; Toichi y Kamio, 2003).

A partir del desempeño observado, las palabras fueron clasificadas por nivel de dificultad según el número de participantes que contestaron correctamente. El rango de sujetos que respondieron correctamente las palabras fluctuó entre 3 a 10. Tres rangos de dificultad (entre 8 a 10, entre 6 a 7 y entre 3 a 5) fueron clasificados como de bajo, medio $\mathrm{y}$ alto nivel de dificultad respectivamente.

\section{Fase 2: Experimento piloto}

\section{Método}

A partir de las palabras graduadas por nivel de dificultad de la Fase 1, se procedió a seleccionar y confeccionar enunciados u oraciones que incorporaran una de las palabras usadas previamente. Dependiendo del tipo de palabra (concreta, abstracta-epistémica o abstracta-metafísica) y su nivel de dificultad (bajo, medio o alto), se diseñaron 9 oraciones. En este experimento se anticipaba que los niños 
con desarrollo típico mostrarían sensibilidad a los niveles de dificultad de las palabras, porque al incrementar la dificultad de las palabras, los aciertos disminuirían y los tiempos de reacción se harían más extensos. En segundo lugar, fue predicho, en términos de efectos principales, que las palabras deberían ser procesadas de manera diferente dependiendo de si eran concretas o abstractas (Toichi y Kamio, 2003). Complementariamente, siguiendo la idea propuesta por Harris y colegas (2006), fue esperado encontrar diferencias en el procesamiento de palabras abstractas-epistémicas y abstractas-metafísicas, bajo el supuesto que si las palabras epistémicas y metafísicas son moduladas por distintos mecanismos de procesamiento semántico, su comportamiento en términos de aciertos y tiempos de reacción debería seguir trayectorias diferentes.

\section{Participantes}

Fueron seleccionados, por disponibilidad, 30 escolares de enseñanza básica provenientes de escuelas de Talca y Peumo, 8 mujeres y 22 hombres, cuyas edades fluctuaron entre los 13 a 15 años $(M=13.3 ; D E=3.81)$.

\section{Materiales y procedimiento}

Los enunciados $\mathrm{u}$ oraciones fueron ensamblados con base a nueve palabras que previamente habían sido graduadas por dificultad. Las palabras concretas fueron cámara, jardín y cristal. Las abstractas-epistémicas fueron expectativas, vergüenza y sospecha. Finalmente, las palabras abstractas-metafísicas fueron seguridad, influencia y desafío (ver Tabla 1).

Tabla 1. Oraciones usadas en la fase experimental.

\begin{tabular}{|c|c|c|c|}
\hline \multirow[b]{2}{*}{ Oraciones incompletas } & \multicolumn{3}{|c|}{ Opciones de Respuesta } \\
\hline & 1 & 2 & 3 \\
\hline $\begin{array}{l}\text { 1. Después de ver la escena del robo, el detective }\left(\_\right) \text {que el ladrón } \\
\text { del diamante puede ser un viejo coleccionista de joyas. [Epis B]. }\end{array}$ & $\underline{\text { Sospecha }}$ & Recuerda & Olvida \\
\hline $\begin{array}{l}\text { 2. El trabajador firmó un contrato por } 10 \text { años con la empresa, lo } \\
\text { que le da más }\left(\_ \text {a su vida y la de su familia. [Meta B]. }\right.\end{array}$ & $\underline{\text { Seguridad }}$ & Confianza & Suspenso \\
\hline $\begin{array}{l}\text { 3. Después de estudiar, el niño decidió salir al ( } \_ \text {para jugar con } \\
\text { su perro y sus amigos. [Conc B]. }\end{array}$ & $\underline{\text { Jardín }}$ & Hospital & Bosque \\
\hline $\begin{array}{l}\text { 4. Cuando se dio cuenta que los demás se reían de él, sintió }\left(\_\right) \text {y } \\
\text { la cara se le puso roja. [Epis } M] \text {. }\end{array}$ & $\underline{\text { Vergüenza }}$ & Angustia & Preocupación \\
\hline $\begin{array}{l}\text { 5. La prueba de matemáticas fue un }(\mathrm{a})(\underset{)}{)} \text { para la inteligencia de } \\
\text { cualquier alumno, realmente era muy difícil. }[\text { Meta } \mathrm{M}] \text {. }\end{array}$ & $\underline{\text { Desafio }}$ & Ventaja & Promesa \\
\hline $\begin{array}{l}\text { 6. Como cada fotografía estaba fuera de foco, el niño concluyó que } \\
\text { la }\left(\_ \text {) estaba dañada y que había que repararla. [Conc } \mathrm{M}\right] \text {. }\end{array}$ & $\underline{\text { Cámara }}$ & Máquina & Tabla \\
\hline 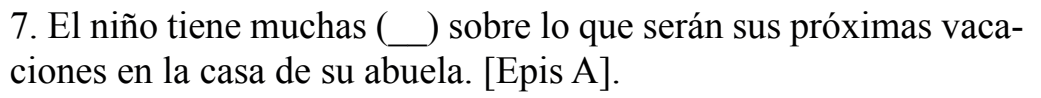 & Expectativas & Preocupaciones & Creencias \\
\hline $\begin{array}{l}\text { 8. Juan cree que su opinión es }\left(\_ \text {) en las decisiones de la empresa }\right. \\
\text { ya que el jefe constantemente le pide su parecer. [Meta A]. }\end{array}$ & Influyente & Oportuna & Genuina \\
\hline 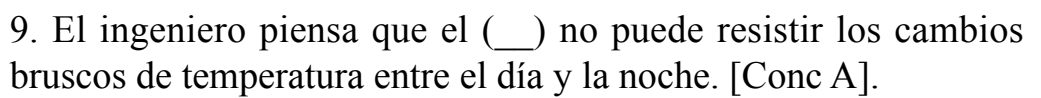 & $\underline{\text { Metal }}$ & Plástico & Cristal \\
\hline
\end{tabular}

Nota: Las oraciones fueron aleatorizadas por el programa E-prime en cada implementación del experimento. Las opciones de respuestas fueron semi-balanceadas durante la programación del experimento, de tal forma que cada opción de respuesta apareciese en la misma proporción a la izquierda, al centro o a la derecha. Esto se hizo con el objetivo de controlar efectos de orden y posición. Sospecha, vergüenza y expectativas corresponden a palabras abstractas-epistémicas (Epis). Seguridad, desafío e influyente corresponden a palabras abstractasmetafísicas (Meta). Finalmente, jardín, cámara y metal corresponden a palabras concretas (Conc). Para cada tipo de palabra hay tres niveles de dificultad: nivel bajo (B), nivel medio (M) y nivel alto (A). 
Los enunciados describen a personas en una situación cotidiana y la palabra a ser completada está en referencia al sujeto y su contexto descrito en el enunciado. Así por ejemplo, en el enunciado "Después de estudiar, el niño decidió salir al (__ para jugar con su perro y sus amigos", la palabra concreta a ser seleccionada (bosque u hospital o jardín) tiene relación con la actividad o verbo que realiza el sujeto en un lugar determinado. Esta misma regla está presente en los enunciados que requerían ser completados con una palabra abstracta-metafísica. Por ejemplo, en el enunciado "Juan cree que su opinión es ( ) en las decisiones de la empresa, ya que el jefe constantemente le pide su parecer", la palabra abstracta-metafísica correcta a ser elegida (genuina o influyente u oportuna) requiere
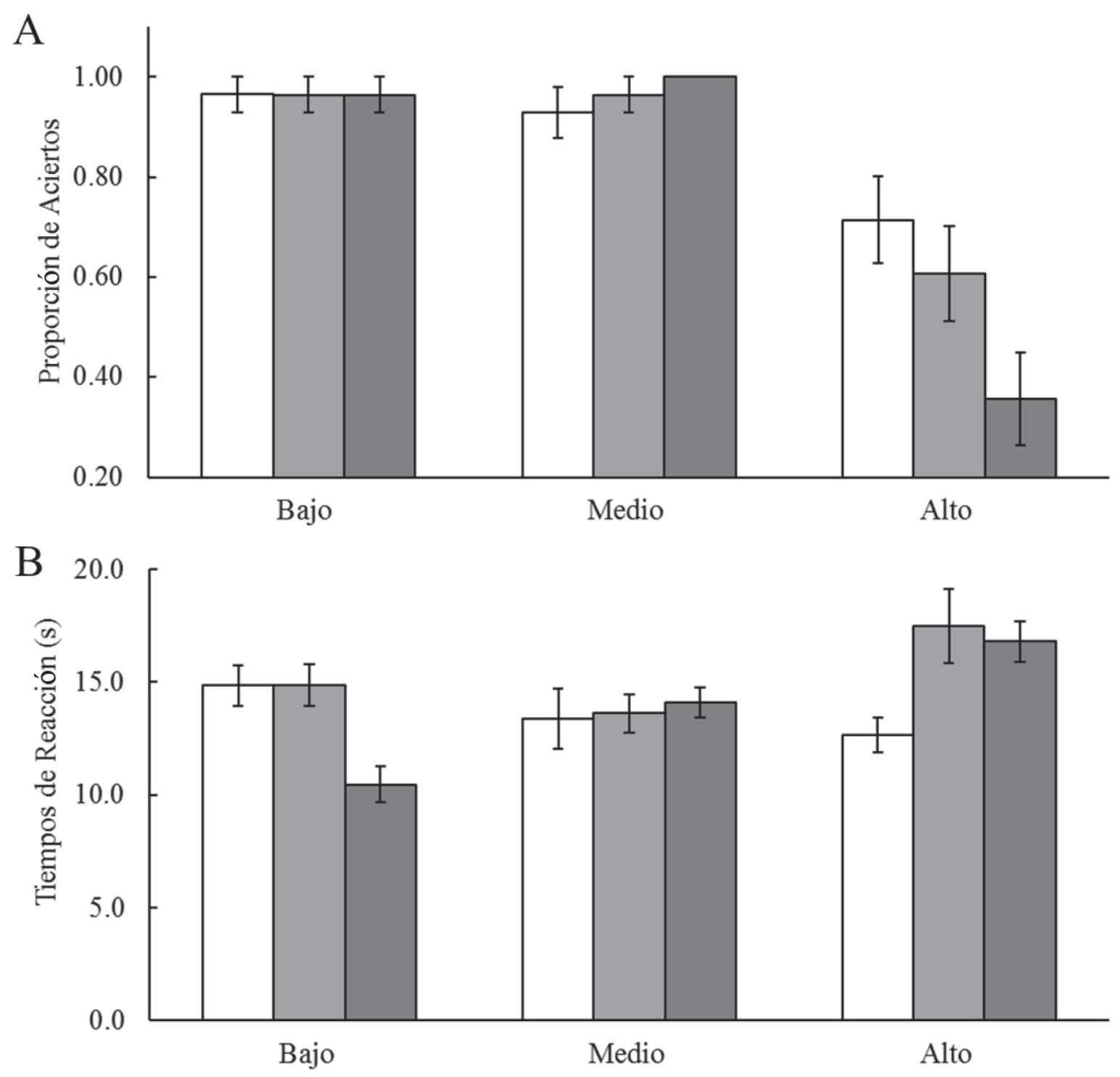

Nivel de Dificultad

\section{$\square$ Abstractas-Epistémicas $\square$ Abstractas-Metafísicas $\square$ Concretas}

Figura 1. Media y errores estándar de la proporción de aciertos (Panel A) y los tiempos de reacción (Panel B) para palabras concretas, abstractas-metafísicas y abstractas-epistémicas graduadas por dificultad en un grupo de niños y adolescentes con desarrollo típico (DT). 
identificar un contexto social (la empresa), el rol causal de las ideas en las decisiones y el estado mental del sujeto, en este caso su creencia. Del mismo modo, con los enunciados que requerían identificar una palabra abstracta-epistémica, la correcta selección se basaba no sólo en identificar el estado mental del sujeto sino que además el contexto en el cual dicho sujeto estaba inserto. En el enunciado "Después de ver la escena del robo, el detective (__ que el ladrón del diamante puede ser un viejo coleccionista de joyas", la palabra abstracta-epistémica obviamente tiene que ver con algún estado mental del sujeto (olvida o sospecha o recuerda). Sin embargo, el estado mental correcto está asociado con el contexto (escena del crimen) en el cual el sujeto desarrolla su trabajo (identificar al autor del robo) y el valor de certeza asociado a la identificación del autor del robo (puede ser).

El experimento fue proyectado en la pantalla del computador, donde aparecía una oración incompleta que el niño debía completar con una palabra. La palabra correcta en conjunto con dos palabras distractoras aparecían como opciones de respuesta en una siguiente imagen. El participante debía elegir una de las tres palabras para completar la oración, presionando en el teclado numérico, el número de la opción de respuesta correspondiente.

El experimento fue dividido en tres fases: La instrucción general, el entrenamiento y el experimento propiamente tal. (a) En la fase de las instrucciones generales aparecía una imagen con la siguiente indicación: "Este es un juego experimental en el computador, que deberás realizar de manera muy cuidadosa. A continuación aparecerán unas oraciones o frases, que deberás leer con atención y completar con la palabra requerida. Primero aparecerá la oración con un espacio en blanco, subrayado con una línea negra y a continuación aparecerán tres palabras y tú deberás seleccionar una de ellas". Después de apretar la barra espaciadora aparecía la siguiente instrucción: "Las palabras tienen en la parte inferior un número y tú deberás apretar la tecla del número que corresponda. A continuación verás un ejemplo". (b) En la fase de entrenamiento era mostrada una imagen con la siguiente oración: "Durante el cumpleaños, el niño sacó dos trozos de (_) porque estaba muy deliciosa".

Inmediatamente aparecía una imagen con las tres palabras como opciones de respuesta, con un número entre paréntesis ubicado debajo de cada palabra: “'Leña (1)', 'Torta (2)' y 'Fruta (3)'”, acompañadas con la siguiente indicación: "Si tú crees que la palabra que mejor se ajusta es 'Leña' aprietas la tecla con el número '1', si crees que es 'Torta' entonces aprietas la tecla con el número '2' y si crees que es 'Fruta' aprietas la tecla con el número '3'. Recuerda que debes apretar una sola tecla. Si estás listo, ahora comienza el juego". (c) Finalmente comenzaba la fase del experimento, la cual partía con alguna de las 9 oraciones detalladas en la Tabla 3.

El orden de presentación de las oraciones fue aleatorizado directamente por el programa para cada participante y el orden de presentación de las opciones de respuesta fue semi-balanceado durante la programación para evitar que la respuesta correcta siempre apareciese en la misma posición. De esta forma, durante el experimento, para tres oraciones, la opción de respuesta correcta apareció a la izquierda (número 1), para otras tres oraciones apareció a la derecha (número 3 ) y finalmente en otras tres oraciones apareció ubicada al medio (número 2). Al emitir la última respuesta aparecía una instrucción en la cual se les agradecía su participación y se les pedía no comentar las respuestas y contenidos del experimento.

El experimento fue programado y administrado con el programa E-prime y duró 5 minutos en promedio, mientras el rango de tiempo para completar la tarea experimental fluctuó entre 3 y 7 minutos. Tanto la rutina experimental como la recolección de las respuestas fueron realizadas usando un computador portátil Packard Bell con un procesador Intel Core Duo y con una pantalla de color de 15,4 pulgadas.

\section{Resultados}

La proporción de respuestas correctas y los tiempos de reacción fueron sometidos a un análisis de varianza de medidas repetidas $3 \times 3$, donde los factores intra-sujetos fueron el nivel de dificultad de las oraciones (alto, medio y bajo) y el tipo de palabras (concretas, abstractas-metafísicas y abstractas-epistémicas).

Los resultados que a continuación son descritos señalan que el procesamiento de palabras insertas en oraciones asume un carácter jerárquico, en el cual las palabras abstractas tienen una mayor proporción de respuestas correctas que palabras concretas. Con palabras de alto nivel de dificultad, las oraciones que deben ser completadas con palabras abstractas-epistémicas tienen más respuestas correctas que las que requieren de palabras concretas. Complementariamente, en términos de tiempos de reacción, las palabras abstractas-epistémicas tienen una tendencia a ser identificadas más rápidamente que las abstractasmetafísicas y concretas. 


\section{Proporción de respuestas correctas}

Fueron detectadas diferencias significativas entre los niveles de dificultad, $F(2.54)=36.29, p<.01, \eta p 2=0.57$, donde la proporción de respuestas correctas de palabras de dificultad baja $(M=0.96)$ y media $(M=0.96)$ fueron significativamente superiores a las palabras de alta dificultad $(M=0.56), p s<.01$ (ver Panel A, Figura 1). En los tipos de palabras fueron encontradas diferencias marginales, $F(2.54)$ $=2.62, p=.08, \eta p 2=0.09$, ya que las palabras abstractasepistémicas $(M=0.87)$ y abstractas-metafísicas $(M=0.85)$ tuvieron promedios de aciertos levemente mayores a los de las palabras concretas $(M=0.77)$.

En tanto, fue detectado un efecto de interacción entre el tipo de palabra y el nivel de dificultad, $F(4.108)=4.25$, $p<0.01, \eta p 2=.14$. Explorando el efecto de interacción al interior de cada nivel de dificultad fue identificado que con bajo y mediano nivel de dificultad no hubo diferencias entre palabras concretas, abstractas-metafísicas y abstractas-epistémicas, $p s>0.48$. Sin embargo, con un alto nivel de dificultad, las abstractas-epistémicas tuvieron una proporción de aciertos significativamente mayor que las palabras concretas, $F(2.26)=4.49, p<.05, \eta p 2=0.26$, no detectándose diferencias significativas entre palabras concretas y abstractas-metafísicas, $p>.15$, o entre los dos tipos de palabras abstractas, $p>.97$. Explorando el efecto de interacción al interior de cada tipo de palabra fue hallado que para las palabras abstractas-metafísicas y concretas, las palabras de mayor dificultad tuvieron un menor número de aciertos que las palabras de nivel bajo y medio de dificultad, $p s<.01$. Entre palabras de nivel medio y bajo de dificultad no fueron detectadas diferencias significativas, $p s>$.97. Para las palabras abstractas-epistémicas, las palabras de mayor dificultad tuvieron una proporción de aciertos menor a las de bajo nivel de dificultad, $p=.05$. Sin embargo, las palabras de nivel medio de dificultad no se diferenciaron de los otros dos tipos de nivel de dificultad, $p s>.17$.

\section{Tiempos de reacción}

Al realizar el análisis de varianza fueron detectados efectos significativos entre los tipos de palabras, $F(2.54)$ $=4.58, p=.02, \eta p 2=0.15$ (ver Panel B, Figura 1), donde las palabras abstractas-metafísicas $(M=15.32 \mathrm{~s})$ tuvieron tiempos de reacción significativamente mayores que las palabras abstractas-epistémicas $(M=13.63 \mathrm{~s}), p=.02$; y marginalmente mayores que las palabras concretas $(M$ $=13.79 \mathrm{~s}), p=.09$. Finalmente no fueron encontradas diferencias entre las palabras concretas y las abstractasepistémicas, $p=.99$.

Fueron halladas diferencias por niveles de dificultad, $F$ $(2.54)=7.37, p=.001, \eta p 2=.21$. Los tiempos de reacción para las palabras de nivel alto de dificultad $(M=15.65 \mathrm{~s})$ fueron significativamente mayores que para las palabras de nivel medio $(M=13.70 \mathrm{~s}), p=.003$; $\mathrm{y}$ nivel bajo $(M=13.39$ s), $p=.01$. Entre los tiempos de reacción de las palabras de nivel medio y bajo de dificultad no fueron detectadas diferencias, $p=.99$.

También fue encontrado un efecto de interacción entre el nivel de dificultad y el tipo de palabra, $F(4.108)=5.85, p$ $<.001, \eta p 2=0.18$. Explorando el efecto de interacción por niveles de dificultad, quedaron en evidencia diferencias con palabras de nivel bajo, $F(2.26)=11.56, p<.001, \eta p 2=$ 0.47 , y de nivel alto de dificultad, $F(2.26)=7.63, p<.01$, $\eta p 2=.37$. Para las palabras de bajo nivel de dificultad, las palabras concretas $(M=12.67 \mathrm{~s})$ y abstractas-metafísicas $(M$ $=13.38 \mathrm{~s})$ tuvieron significativamente menores tiempos de reacción que las palabras abstractas-epistémicas $(M=14.84$ $\mathrm{s}), p \mathrm{~s}<.01$; mientras que los tiempos de reacción entre las palabras concretas y abstractas-metafísicas no fueron distintos entre sí, $p=.99$. En el caso de las palabras clasificadas de alto nivel de dificultad, las palabras abstractas-epistémicas tuvieron tiempos de reacción significativamente menores $(M=10.45 \mathrm{~s})$ que las palabras concretas $(M=16.81 \mathrm{~s}), p=$ .002 ; y que las palabras abstractas-metafísicas $(M=14.12$ s), $p=.02$. En tanto, los tiempos de reacción entre palabras concretas y abstractas-metafísicas no fueron diferentes, $p$ $=.99$.

La exploración del efecto de interacción en cada tipo de palabras dejo en evidencia para las palabras concretas los tiempos de reacción con altos niveles de dificultad ( $M$ $=16.81 \mathrm{~s}$ ) fueron marginalmente mayores que con niveles medios $(M=14.12 \mathrm{~s}), p=.06$; $\mathrm{y}$ significativamente mayores que con niveles bajos de dificultad $(M=10.45 \mathrm{~s}), p<.001$. A su vez, los niveles medios tomaron más tiempo en ser respondidos que los niveles bajos de dificultad, $p=.02$. Para las palabras abstractas-epistémicas no hubo diferencias entre los niveles de dificultad bajo $(M=14.84 \mathrm{~s})$, medio $(M$ $=13.38 \mathrm{~s}) \mathrm{y}$ alto $(M=12.67 \mathrm{~s}), p s>.19$. Tampoco para las palabras abstractas-metafísicas hubo diferencias entre los niveles de dificultad bajo $(M=14.88 \mathrm{~s})$, medio $(M=13.62$ s) y alto $(M=17.47 \mathrm{~s}), p s>.07$. 


\section{Discusión}

Los objetivos de este experimento piloto estuvieron orientados a evaluar la sensibilidad de los niveles de dificultad de las palabras y someter a contraste las hipótesis que establecían que las palabras abstractas en general eran procesadas de manera diferente a las palabras concretas, y que las palabras abstractas-epistémicas deberían ser más rápidas de procesar y con más aciertos que las abstractas-metafísicas.

Los resultados en general mostraron que las diferencias por niveles de dificultad fueron más notorias entre los extremos. Es decir, las oraciones que requerían identificar palabras con altos niveles de dificultad tuvieron tiempos de reacción mayores y proporciones de aciertos menores que las palabras con niveles bajos de dificultad. Sin embargo, las palabras de nivel medio de dificultad resultaron muy parecidas a las de nivel bajo y en varios contrastes tampoco se diferenciaron de las palabras de nivel alto de dificultad. La diferencia entre altos y bajos niveles de dificultad también fue observada entre los tipos de palabras, aun cuando esta tendencia fue más clara para las palabras concretas.

En segundo lugar, en términos de efectos principales fue esperado encontrar que las palabras abstractas (epistémicas y metafísicas) fueran diferentes a las concretas. Los resultados señalaron que las palabras abstractas en general obtuvieron levemente una mayor proporción de aciertos que las palabras concretas. Complementariamente, sólo las palabras abstractas-metafísicas tomaron marginalmente más tiempo en ser respondidas que las concretas, porque las palabras concretas y abstractas-epistémicas fueron igualmente rápidas al ser procesadas. Creemos que esto se debe principalmente a que la oración en la cual está inserta una palabra facilita un procesamiento semántico. Los estudios preliminares muestran que las palabras concretas son recordadas o imaginadas de mejor manera que las abstractas (Toichi y Kamio, 2003). Eso fue hallado en la Fase 1 de este estudio. Los sujetos observaron palabras aisladas en la pantalla de un computador y proporcionaron mejores definiciones para las palabras concretas que para las palabras abstractas. Empero, en este experimento piloto, las palabras en vez de ser presentadas aisladamente fueron insertadas en una oración. Suponemos que la lectura de la oración incompleta promovió un procesamiento semántico más profundo que la simple evocación de significado de una palabra aislada (Toichi y Kamio, 2002). Aparentemente, las oraciones que nosotros creamos, en la medida que incorporaron un sujeto y una acción en un contexto social, afectaron positivamente el desempeño en la identificación de palabras abstractas, mientras que con las palabras concretas su impacto fue menor.

Finalmente, los resultados fueron parcialmente coincidentes con la hipótesis que proponía que las palabras abstractasepistémicas serían procesadas con mayor velocidad y con más aciertos que las palabras abstractas-metafísicas. Esta hipótesis fue propuesta tomando en cuenta los hallazgos de Harris y colaboradores (2006). Ellos detectaron que en el procesamiento de palabras presentadas aisladamente, las epistémicas fueron marginalmente más veloces y con menos valoraciones positivas que las palabras metafísicas, bajo el supuesto que si las palabras epistémicas y abstractas son moduladas por distintos mecanismos de procesamiento semántico, su comportamiento debería seguir trayectorias diferentes.

En este experimento piloto ambas palabras fueron similares en términos de la proporción de aciertos, y esta tendencia se mantuvo en los tres niveles de dificultad. Sólo en términos de tiempos de reacción se pudo establecer que las palabras abstractas-epistémicas tomaron menos tiempo en ser procesadas que las abstractas-metafísicas y concretas, coincidente con Harris y su grupo. Aun cuando esta tendencia sólo fue observada en altos y bajos niveles de dificultad -porque en los niveles medios de dificultad ambos tipos de palabras fueron similares en velocidad-, las palabras abstractas-epistémicas tomaron más tiempo en ser respondidas que las abstractas-metafísicas.

Con estos antecedentes podemos afirmar que el procesamiento de palabras graduadas en dificultad e insertas en oraciones, llevado a cabo por niños con desarrollo típico (DT), sigue un patrón jerárquico en el cual las palabras abstractas tienen tendencia a una mayor proporción de aciertos que las palabras concretas, donde las oraciones que requieren de palabras abstractas-epistémicas tienen mayores aciertos que las que requieren de palabras concretas, cuando son usadas palabras de alto nivel de dificultad. Concordantemente, al analizar la velocidad de procesamiento es posible observar una tendencia de las palabras abstractas-epistémicas a tomar menos tiempo que las abstractas-metafísicas concretas. Sin embargo, un intrincado patrón de interacción indica que con bajos, medios y altos niveles de dificultad, las palabras abstractas-metafísicas y concretas demoran lo mismo, mientras que las abstractas-epistémicas toman más y menos tiempo que las concretas con bajos y altos niveles de dificultad respectivamente. 


\section{Experimento final}

\section{Método}

A partir de los hallazgos de la Fase 2, se procedió a implementar el experimento final. En esta oportunidad, el objetivo fue comparar el procesamiento de palabras abstractas (metafísicas y epistémicas) y concretas en un grupo de niños y adolescentes con trastornos de espectro autista (TEA), igualado con un grupo de niños y adolescentes con desarrollo típico (DT). La igualación fue una a una en variables sociodemográficas tales como género y edad, además de las funciones cognitivas e intelectuales medidas a través de las pruebas de inteligencia de Wechsler: WAIS y WISC-III.

La hipótesis general apuntaba a que los niños con TEA tendrían una menor proporción de aciertos y mayores tiempos de reacción en palabras abstractas que concretas que los niños con DT. En la misma línea argumental, fue predicho que en las palabras abstractas-epistémicas este bajo desempeño sería mucho más acentuado. Finalmente, bajo el supuesto que el contexto de la oración mejora el desempeño en la identificación de las palabras abstractas,

Tabla 2. Comparación del funcionamiento intelectual de los niños y adolescentes con trastornos de espectro autista (TEA) $y$ con desarrollo típico (DT), en las escalas Wechsler (WAIS y WISC-III).

\begin{tabular}{lll}
\hline Sub-escalas y escalas & $\begin{array}{l}\text { TEA }(\mathrm{n}=16) \\
\text { Media }(D E)\end{array}$ & $\begin{array}{l}\text { DT }(\mathrm{n}=16) \\
\text { Media }(D E)\end{array}$ \\
\hline Completación de figuras & $9.90(2.1)$ & $9.70(1.9)$ \\
Información & $9.60(2.8)$ & $9.20(2.9)$ \\
Claves & $8.10(2.4)$ & $8.50(2.3)$ \\
Analogías & $9.30(1.7)$ & $9.10(1.9)$ \\
Ordenamiento de historias & $9.90(2.6)$ & $9.60(2.1)$ \\
Aritmética & $9.60(3.4)$ & $9.40(3.0)$ \\
Construcción con cubos & $11.40(4.0)$ & $11.00(2.8)$ \\
Vocabulario & $7.80(2.1)$ & $8.10(2.2)$ \\
Ensamblaje de objetos & $8.60(1.9)$ & $9.00(1.8)$ \\
Comprensión & $5.70(2.8)$ & $6.80(2.1) *$ \\
Escala Verbal & $91.10(9.4)$ & $91.40(9.3)$ \\
Escala Ejecución & $97.50(10.4)$ & $97.10(10.0)$ \\
Escala total & $93.20(8.4)$ & $93.40(8.2)$ \\
\hline
\end{tabular}

*El funcionamiento intelectual medido con las escalas Wechsler es similar entre sujetos con TEA y con DT. Sin embargo, en la sub-escala de comprensión, los sujetos con TEA tienen significativamente menos puntaje que los sujetos con DT, $p<.05$. fue anticipado que los niños con TEA serían menos sensibles a estas claves, comparados con el grupo de pares con DT.

\section{Participantes}

El grupo experimental estuvo constituido por 16 niños y adolescentes diagnosticados con TEA, que asistían a colegios con proyectos de integración, con una media en edad cronológica de 14.6 años, 5 mujeres y 11 hombres, cuyo coeficiente intelectual promedio fue de 93.2 puntos. Para conformar el grupo control se evaluó a 64 niños y adolescentes con desarrollo típico (DT), a partir de los cuales

Tabla 3. Caracterización de los niños y adolescentes con TEA a partir del reporte de los padres y/o cuidadores con la Childhood Autism Rating Scale (C.A.R.S.).

Características $\%$

Para cambiar las rutinas hay que avisarle con anticipación y explicarle bien.

No presenta ecolalia.

Antes de los 3 años presentó un funcionamiento anormal en curiosidad frente al ambiente.

Ante los olores, sabores y texturas, hace notar su agrado o desagrado en forma notoria.

No soporta estar mucho tiempo quieto.

Antes de los 3 años presentó un funcionamiento anormal en lenguaje.

Presenta interés en juguetes u objetos. $\quad 75$

Los sonidos fuertes y desconocidos lo alteran y asustan. $\quad 75$

Presenta un área académica más marcada que otras. $\quad 75$

Antes de los 3 años presentó un funcionamiento anormal en interacción social.

Antes de los 3 años presentó un funcionamiento anormal 75 en juego simbólico.

Comparte con los demás. $\quad 72$

Su atención es breve y no se comunica mirando a los ojos.. 68

Comprende los gestos pero tiende a malinterpretarlos. $\quad 68$

La coordinación de los movimientos corporales es torpe.. 62

No imita.

Ante un examen y reuniones sociales se pone ansioso y 53 no soporta los cambios del entorno.

Presenta reciprocidad emocional. 12

Nota: Sólo los padres de niños con TEA contestaron esta escala. La frecuencia de los puntajes en los síntomas descritos por el instrumento, coincide con el diagnóstico de trastorno de espectro autismo que los niños han recibido previamente. 
se eligieron 16 que se correspondieron más fielmente con sus pares del grupo con TEA. Los alumnos de este grupo asistían a colegios de las ciudades de Rancagua y Peumo, con una media de edad cronológica de 14,6 años, cuyo coeficiente intelectual promedio fue de 93.4 (Tabla 2). Aun cuando ambos grupos fueron igualados en el CI global, CI verbal y CI manual, el grupo de niños con TEA tuvo significativamente menores puntajes que el grupo control en la sub-escala de comprensión, $t(15)=2.70, p=.02$, la cual evalúa la capacidad del sujeto para comprender e interiorizar las costumbres, situaciones, normas y prácticas sociales. En los análisis posteriores, esta variable es incluida como covariada, con el objetivo de igualar estadísticamente ambos grupos.

\section{Materiales e instrumentos}

La Escala de Autismo Infantil (Childhood Autism Rating Scale, C.A.R.S.) de Schopler, Reichler y Renner (1988) es una escala de apreciación conductual cuyo objetivo es el diagnóstico y la planificación del tratamiento de sujetos con autismo y ayuda a diferenciar a los niños con autismo de los que tienen otros retrasos en el desarrollo. Cuenta con una confiabilidad de 0,94 y validez de criterio de 0,80 . Para efectos de este estudio, la escala fue traducida y adaptada verbalmente para una mejor comprensión por parte de los apoderados, ya que el objetivo de su uso no era el de diagnosticar a los niños y adolescentes (Tabla 3). Esta adaptación ad-hoc no fue sometida a un proceso de validación de jueces y se usó sólo con el fin de caracterizar la muestra clínica que ya había sido diagnosticada previamente por médicos y psicólogos.

Wechsler Adult Intelligence Scale (WAIS) y Wechsler Intelligence Scale for Children (WISC-III) (Wechsler, 1999). Las pruebas tienen las siguientes sub-escalas: información, comprensión, aritmética, semejanzas (analogía en WISCIII), vocabulario, claves, completación, cubos, ordenación y ensamblaje. Las primeras 5 sub-escalas conforman la escala verbal y las otras 5 conforman la escala de ejecución. Ambas escalas dan un puntaje total, el cual es considerado como coeficiente intelectual. Ambas pruebas están adaptadas y cuentan con normas para la población chilena (Hermosilla y Valencia, 1982; Ramírez y Rosas, 2007).

\section{Procedimiento}

Para conformar la muestra de sujetos con TEA se realizó un catastro de los colegios con proyecto de integración y se procedió a seleccionar a alumnos con trastorno autista o trastorno de Asperger, descartando a niños y adolescentes con déficit intelectual severo y otros trastornos del desarrollo. Esta selección fue hecha revisando los informes psicológicos y médicos de cada potencial participante, previa autorización de los padres. Después de identificar a los sujetos pre-seleccionados se entregó un consentimiento informado a sus respectivos apoderados (con DT y con TEA). Luego se procedió a entregar la pauta de observación conductual sólo a los padres de niños y adolescentes con TEA.

Uno de los investigadores se reunió con cada sujeto antes de la aplicación de la prueba de inteligencia y la implementación del experimento, con el fin de generar una primera aproximación. Esa reunión fue individual y se realizó en una sala del establecimiento educativo. Luego de establecer contacto con los participantes, se procedió a aplicar la prueba de inteligencia a los sujetos con TEA en sus respectivos establecimientos, la cual demoró en promedio 75 minutos, distribuidos en dos sesiones. Las evaluaciones fueron llevadas a cabo en una sala aislada de ruidos y objetos distractores. A medida que fue completada la evaluación del desempeño intelectual de los niños y adolescentes con TEA, se evaluó a sus pares con DT, en una proporción de 4 sujetos con DT por cada sujeto con TEA, antes de encontrar su par más próximo. Una vez evaluado el desempeño intelectual se procedió a aplicar el experimento a todos los participantes seleccionados. El experimento fue idéntico al aplicado en la fase 2, programado con E-prime e implementado en un computador portátil Packard Bell con un procesador Intel Core Duo y con una pantalla de color de 15.4 pulgadas.

\section{Resultados}

La proporción de respuestas correctas y los tiempos de reacción fueron sometidos a un análisis de covarianza mixto $2 \times 3 \times 3$, donde el factor inter-sujetos fue el grupo con TEA y el grupo con DT, mientras que los factores intra-sujetos fueron el nivel de dificultad de las oraciones (alto, medio y bajo) y el tipo de palabras (concretas, abstractas-metafísicas y abstractas-epistémicas). Para controlar las diferencias observadas en la sub-escala de comprensión, esta variable fue incluida como una covariada. De aquí en adelante, las medias reportadas corresponden a las medias estimadas, cuando el efecto de la variable comprensión ha sido estadísticamente controlado o equiparado entre ambos grupos.

En términos globales, los resultados que a continuación son descritos muestran que los sujetos con TEA tuvieron más dificultades en el procesamiento de palabras abstractasepistémicas que con palabras abstractas-metafísicas (Figura 

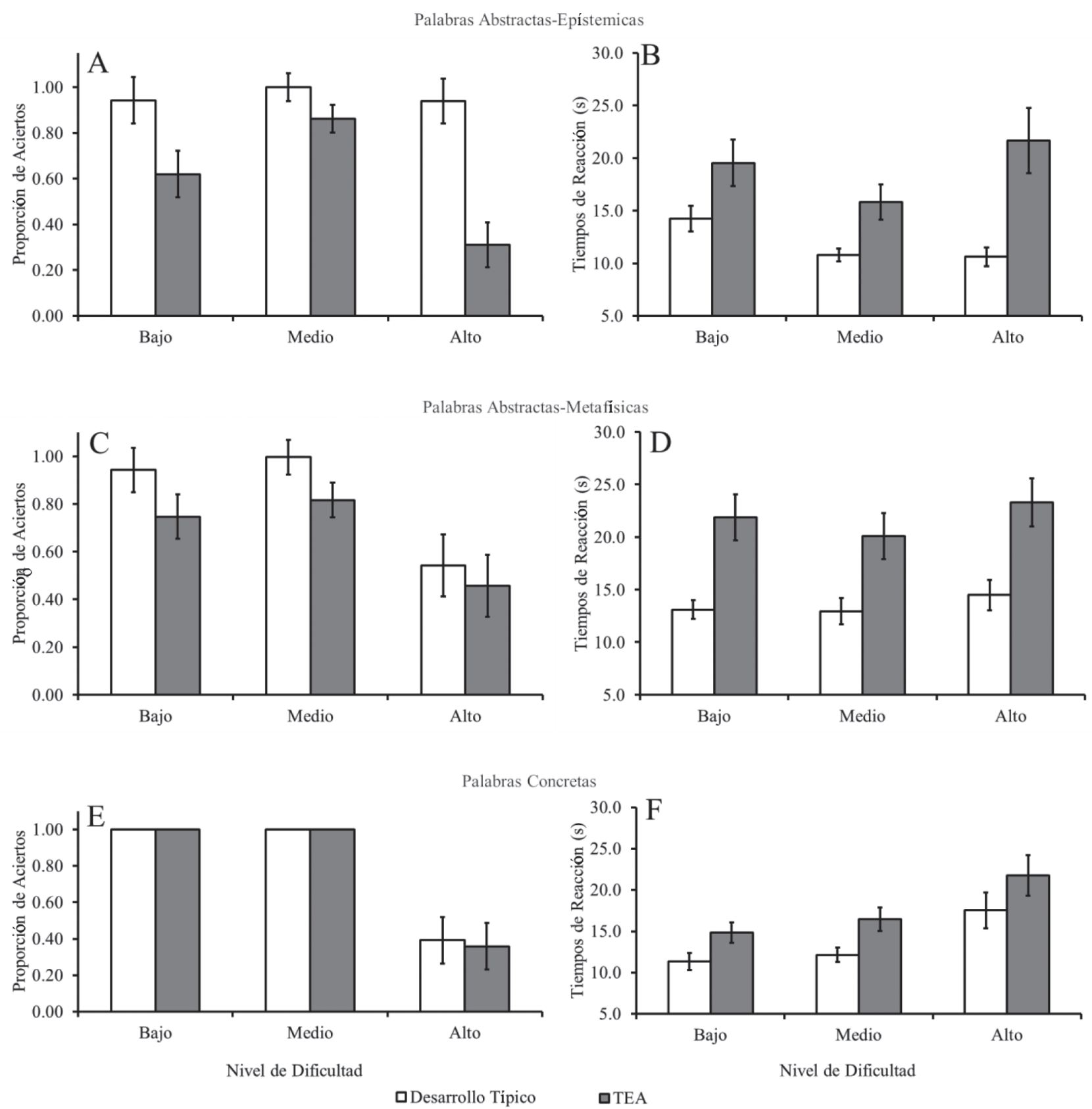

Figura 2. Medias de porcentaje de respuestas correctas (paneles A, C y E) y los tiempos de reacción (paneles $B, D$ y F) en las muestras de niños y adolescentes con Trastorno de Espectro Autista (TEA) y con Desarrollo Típico (DT), para las palabras abstractas epistémicas (A-B), abstractas metafisicas $(C-D)$ y palabras concretas $(E-F)$.

2). Estos resultados no pueden ser atribuidos al nivel de dificultad, porque dicha variable fue controlada. Por lo tanto, los hallazgos tienen que ver exclusivamente con el tipo de palabra. Si una variable menos sensible, como la proporción de respuestas correctas, detectó una diferencia en las palabras abstractas-epistémicas y no para las abstractas-metafísicas, podemos concluir que las palabras abstractas-epistémicas fueron más difíciles de procesar para el grupo con TEA que para el grupo con DT.

\section{Proporción de respuestas correctas}

Fueron detectadas diferencias entre los grupos (ver Figura 2, paneles A, C y E), donde los sujetos con TEA tuvieron una proporción de aciertos menor $(M=0.69)$ que los sujetos con DT $(M=0.86), F(1.58)=24.1, p<$ $.001, \eta p 2=0.45$. Esta tendencia se mantuvo en todos los niveles de dificultad, ya que los niños con TEA tuvieron un desempeño significativamente menor con palabras de bajo 
nivel de dificultad $(M T E A=0.79$ versus $M D T=0.96), F$ $(1.29)=6.21, p=.02, \eta p 2=0.18$; con palabras de nivel medio $(M T E A=0.89$ versus $M D T=1.00), F(1.29)=7.17$, $p=.01, \eta p 2=0.20$; $\mathrm{y}$ finalmente en palabras de niveles altos de dificultad $(M T E A=0.38$ versus $M D T=0.63), F(1.29)$ $=9.14, p=.001, \eta p 2=0.24$.

Independientemente del grupo y del tipo de palabra, fueron detectadas diferencias entre los niveles de dificultad, $F(2.58)=7.98, p=.001, \eta p 2=0.22$, donde las de nivel bajo $(M=0.88)$ y medio de dificultad $(M=0.95)$ obtuvieron una proporción de aciertos mayores que las palabras de alto nivel de dificultad $(M=0.50), p<.001$, mientras que entre palabras de nivel bajo y medio de dificultad no hubo diferencias significativas, $p>.36$. No fueron halladas diferencias significativas entre palabras abstractas-epistémicas $(M=0.78)$, abstractas-metafísicas $(M=0.75)$ y palabras concretas $(M=0.79), F(1.58)=1.16, p=.32$.

Finalmente se encontró una interacción significativa entre el tipo de palabra y el grupo, $F(2.58)=6.02, p<$ $.01, \eta p 2=0.17$. Explorando esta interacción al interior de cada tipo de palabra, los niños con TEA tuvieron una proporción significativamente menor de respuestas correctas que los niños con DT con palabras abstractas-epistémicas $(M T E A=0.60$ versus $M D T=0.97), F(1.29)=33.84, p$ $<.001, \eta p 2=0.54$; y marginalmente menor en palabras abstractas-metafísicas (MTEA $=0.67$ versus $M D T=0.83)$, $F(1.29)=3.37, p=.07, \eta p 2=.08$. En tanto, en las palabras concretas ambos grupos tuvieron prácticamente el mismo promedio de aciertos $(M T E A=0.79$ versus $M D T$ $=0.80), p>.85$. Explorando la interacción al interior de cada grupo, fue posible detectar que los niños con TEA tuvieron una proporción mayor de respuestas correctas con palabras concretas $(M=0.79)$ que con palabras abstractasepistémicas $(M=0.60), p=.02$, mientras que las palabras abstractas-metafísicas $(M=0.67)$ no se diferenciaron de los demás tipos de palabras, $p s>$.47. Para los niños con DT, la proporción mayor de aciertos fue marginalmente significativa con las palabras abstractas-epistémicas $(M=0.97)$ que con las concretas $(M=0.80), p=.051$, no diferenciándose las palabras abstractas-metafísicas $(M=0.83)$ de los demás tipos de palabras, $p s>.19$.

La sub-escala de comprensión, como covariada, no resultó estadísticamente significativa por sí misma, $p>$ $.64, \mathrm{y}$ tampoco interactuó significativamente con otras variables, $p s>.42$.

\section{Tiempos de reacción}

Fueron detectadas diferencias en los tiempos de reacción (ver Figura 2, paneles B, D y F), donde los niños con TEA tomaron más tiempo en responder $(M=19.33 \mathrm{~s})$ que los niños con DT $(M=13.17 \mathrm{~s}), F(1.29)=11.36, p<.01$, $\eta p 2=0.28$. Esta tendencia se mantuvo en las palabras abstractas-epistémicas $(M T E A=18.87 \mathrm{~s}$ versus $M D T=$ $12.03 \mathrm{~s})$, abstractas-metafísicas $(M T E A=21.58 \mathrm{~s}$ versus $M D T=13.66 \mathrm{~s}), \mathrm{y}$ concretas $(M T E A=17.55 \mathrm{~s}$ versus $M D T$ $=13.83 \mathrm{~s}), F_{s}(1,29) \geq 4.48, p s<.05, \eta p 2 \geq 0.13$.

También fueron encontradas diferencias por tipos de palabras, $F(2.28)=7.79, p<.001, \eta p 2=0.36$. Independiente del grupo y el nivel de dificultad de la palabra, los sujetos tomaron significativamente más tiempo con las palabras abstractas-metafísicas ( $M=17.62 \mathrm{~s})$, que con las palabras abstractas-epistémicas $(M=15.45 \mathrm{~s}), p=.003$; y marginalmente más tiempo que con palabras concretas $(M=15.69$ s), $p=.07$. A su vez, entre palabras abstractas-epistémicas $\mathrm{y}$ concretas no fueron detectadas diferencias significativas, $p=.99$.

Por nivel de dificultad, independiente del grupo y el tipo de palabra, también fueron detectadas diferencias, $F(2.28)$ $=6.60, p<.01, \eta p 2=.32$, donde los sujetos tomaron más tiempo contestando palabras de alto nivel de dificultad $(M$ $=18.23 \mathrm{~s})$ que palabras de nivel medio $(M=14.71 \mathrm{~s}), p=$ .003 , y de nivel bajo $(M=15.83 \mathrm{~s}), p=.03$. Mientras que entre palabras de nivel bajo y nivel medio de dificultad no hubo diferencias significativas, $p>.57$.

Fue hallada una interacción entre el tipo de palabra y el grupo, $F(2.58)=3.88, p<.05, \eta p 2=0.12$. En los sujetos con DT, los tiempos de reacción entre los tipos de palabras fluctuaron entre $12.03 \mathrm{~s}$ y $13.83 \mathrm{~s}$, con ninguna diferencia significativa entre ellos, $p \mathrm{~s}>.18$. En cambio, los niños con TEA tomaron más tiempo con las palabras abstractasmetafísicas $(M=21.58 \mathrm{~s})$, que con las palabras concretas $(M=17.55 \mathrm{~s}), p<.01$, y que con las palabras abstractasepistémicas $(M=18.87 \mathrm{~s}), p<.01$. Sin embargo, entre las palabras concretas y abstractas-epistémicas, los tiempos de reacción no fueron significativamente distintos, $p>.89$.

Aun cuando entre el nivel de dificultad y el grupo no hubo una interacción significativa, se realizaron comparaciones pareadas entre los niveles de dificultad al interior de cada grupo. En los niños con DT, los tiempos de reacción de los niveles de dificultad fluctuaron entre 12.08 s y 14.30 
$\mathrm{s}$, con ninguna diferencia significativa entre ellos, $p \mathrm{~s}>.36$. En cambio, los niños con TEA tomaron más tiempo con las palabras de nivel alto de dificultad $(M=22.15 \mathrm{~s})$, que con las de nivel medio de dificultad ( $M=17.33 \mathrm{~s}), p<.01$, y que con las palabras de nivel bajo de dificultad $(M=18.52$ s), $p<.03$. Sin embargo, entre las palabras de nivel medio y nivel bajo de dificultad, los tiempos de reacción no fueron significativamente distintos, $p>.98$.

La sub-escala de comprensión no resultó estadísticamente significativa por sí misma, $p>.47$; y tampoco interactuó significativamente con otras variables del modelo, $p s>.58$.

\section{Discusión general}

El experimento final fue diseñado para demostrar que los niños con TEA, con el mismo nivel de funcionamiento intelectual que los niños con DT, tienen un procesamiento claramente deficitario en palabras abstractas-epistémicas. Aun cuando los niveles de concreción e imaginabilidad de las palabras abstractas-metafísicas y abstractas-epistémicas son similarmente bajos, existe bastante evidencia como para asumir que las palabras epistémicas y metafísicas tienen trayectorias de desarrollo distintas que les imprimen una modalidad de procesamiento diferente. A nuestro saber, las primeras estarían más conectadas a la coordinación temprana entre los estados internos del niño, la detección de miradas y la atención conjunta. Aspectos claves en el uso de todas las palabras, pero que en el caso de las palabras epistémicas cobran más relevancia dado que son componentes básicos que regulan la interacción social (Charman et al., 2000; Liebal et al., 2009; Tomasello, 1996; Tirassa et al., 2006).

Existe una larga lista de investigaciones que demuestra que las personas con autismo y con síndrome de Asperger de alto funcionamiento intelectual poseen un déficit marcado para entender los estados mentales de los otros (Baron-Cohen et al., 1985, 1986 y 1994; Happé, 1993 y 1995; Loukusa et al., 2007). Desde tempranas etapas desarrollo, estos niños tienen dificultades para capturar la intencionalidad de los otros (Tager-Flusberg, 1992; Ziatas et al., 1998). Este déficit no sólo está circunscrito al área del razonamiento con estados mentales, sino que se extiende a procesos de memoria, de lenguaje y de la comprensión de lectura (Brown et al., 2013; Tesink et al., 2011). Por ejemplo, es reconocida la dificultad de los niños con TEA para diferenciar verbos que refieren acciones concretas de verbos que refieren estados mentales (Baron-Cohen et al., 1994). También ha sido confirmado que las personas con TEA de alto funcionamiento intelectual no tienen dificultades en interpretar la ambigüedad de las palabras, sino que el problema emerge cuando para desambiguar significados es necesario integrar claves sociales o el punto de vista de los otros (Dennis et al., 2001). La comprensión de textos tampoco es dificultosa para las personas con TEA, con la excepción de párrafos o enunciados que aluden a un conocimiento de cómo funciona el entorno social (Brown et al., 2013). Si estos resultados son resumidos, podemos decir que el factor común a este déficit tiene que ver con integrar la intencionalidad presente en el contexto social.

Existe otra línea de investigación, orientada a explorar las diferencias de procesamiento semántico y perceptual y su relación con los mecanismos de memoria. Hasta ahora ha sido demostrado que las personas con TEA mantienen el procesamiento perceptual y semántico al mismo nivel, sin la jerarquía a menudo observada en personas con DT, en la cual lo semántico está por encima de lo perceptual (Toichi y Kamio, 2002). También es conocido que para las personas con TEA, la velocidad y acuciosidad de las palabras concretas está al mismo nivel que las palabras abstractas, mientras que en personas con DT, las palabras concretas son procesadas más eficientemente que las abstractas (Toichi y Kamio, 2003).

Siguiendo esta línea de trabajo, nosotros profundizamos más en la explicación de las diferencias de procesamiento de palabras insertas en enunciados. Para eso seleccionamos y calibramos, por nivel de dificultad, palabras que aluden a estados epistémicos, entidades metafísicas y objetos concretos. Además insertamos estas palabras en oraciones que describen las acciones de un sujeto en un contexto social. Esfuerzos preliminares por encontrar diferencias en el desempeño y la activación de áreas cerebrales para este tipo de palabras (concretas, epistémicas y metafísicas) no habían sido del todo satisfactorios (Harris et al., 2006).

Una de las hipótesis bajo escrutinio proponía que si hubiese una fuente común de procesamiento para las palabras abstractas, deberíamos esperar que el desempeño en palabras epistémicas y metafísicas fuese similar, sin ninguna distinción entre ellas. Y si los sujetos con TEA tienen una alteración en esta fuente de procesamiento (la atención conjunta y la captación de intencionalidad), que hace que las palabras abstractas sean adquiridas tardíamente y usadas con dificultad, entonces el déficit debería expresarse homogéneamente en ambos tipos de palabras. Sin embargo, sí es aceptada la hipótesis que el procesamiento de palabras abstractas es heterogéneo en su naturaleza, es decir, las palabras epistémicas que aluden a estados mentales tienen una modalidad de procesamiento distinta a las metafísicas, donde el procesamiento de palabras abstractas-epistémicas 
no estaría del todo conectado al funcionamiento intelectual como sí lo estaría el procesamiento de las palabras abstractasmetafísicas; entonces deberíamos esperar que los sujetos con TEA de alto nivel intelectual entendieran y usaran las palabras abstractas, con excepción de aquellas que aluden a estados mentales, es decir, las abstractas-epistémicas.

Los hallazgos muestran que los sujetos con TEA, ante oraciones que requieren ser completadas con palabras abstractas epistémicas, cometen significativamente más errores que los sujetos con DT. Esta tendencia también se observó marginalmente para las palabras abstractas-metafísicas. Del mismo modo, el análisis de interacción señaló que los sujetos con TEA cometieron más errores con las palabras epistémicas que con las concretas, mientras que los sujetos con DT la tendencia fue opuesta, ya que cometieron más errores con palabras concretas que con palabras epistémicas. En este caso conjeturamos que el contexto social de la oración (la presencia de un sujeto y un complemento) en el cual estaba inserta la palabra, afectó diferencialmente el desempeño de los sujetos. Mientras el contexto de la oración, que promueve un procesamiento semántico, facilitaría la interpretación de palabras epistémicas, al mismo tiempo podría tener un efecto deletéreo o de interferencia en la interpretación de las palabras concretas. Visto de esta manera, dicho efecto contextual condujo a los sujetos con DT a identificar correctamente las palabras epistémicas, mientras que interfirió en la identificación de las palabras concretas. Por el contrario, en los sujetos con TEA, la insensibilidad a las claves sociales presentes en la oración no los ayudó a identificar las palabras epistémicas y tampoco los interfirió en la identificación de las palabras concretas.

Cuando son analizados los tiempos de reacción es posible apreciar que los sujetos con TEA fueron más lentos para responder que los sujetos con DT. Independiente del grupo, las palabras abstractas-metafísicas tomaron más tiempo que las abstractas-epistémicas y concretas. Además, los efectos de interacción mostraron que los sujetos con DT fueron igualmente rápidos en términos de velocidad, independientemente del tipo de palabra y del nivel de dificultad, mientras que los sujetos con TEA tomaron más tiempo con palabras abstractas-metafísicas y fueron sensibles a la manipulación de los niveles de dificultad. De esta forma los efectos principales observados se debieron básicamente al desempeño de los sujetos con TEA. Estos resultados estarían indicando que el contexto social de la oración hace más lenta la identificación de la palabra correcta solo, en los sujetos con TEA, y que esta interferencia se hace más notoria con palabras abstractas-metafísicas, las cuales suponemos requieren de un componente intelectual mayor que las epistémicas cuando deben ser procesadas.

Si los tiempos de reacción y la proporción de respuestas correctas son mirados en su conjunto es posible establecer que los sujetos con TEA tuvieron más dificultades en el procesamiento de palabras abstractas-epistémicas que con palabras abstractas-metafísicas. En el procesamiento de palabras abstractas-epistémicas, ambas variables dependientes convergieron en señalar esta diferencia. En cambio, para las palabras abstractas-metafísicas las diferencias entre ambos grupos fueron observadas sólo para los tiempos de reacción.

Un elemento que potencialmente podría haber explicado las diferencias observadas en nuestro experimento fueron las diferencias en las puntuaciones de la sub-escala de comprensión de la escalas Wechsler. Como fue descrito en el procedimiento, los participantes de este experimento fueron evaluados con las pruebas de Wechsler para igualarlos en su nivel de funcionamiento intelectual. Con la idea de hacer un pareo exacto fue necesario evaluar a 4 sujetos con DT por cada sujeto con TEA (4:1). Aun cuando el pareo obtenido en el diseño permitió igualar ambos grupos en los puntajes totales, los niños y adolescentes con TEA presentaron puntajes significativamente inferiores en la sub-escala de comprensión, lo que es consistente con estudios previos, los cuales han demostrado que los sujetos con TEA de alto nivel intelectual tienen problemas para comprender guiones sociales en los que la captura de la intencionalidad de las acciones es más importante que su coherencia lógica (Dennis et al., 2001). En este contexto, aun cuando nosotros no fuimos capaces de homologar perfectamente en el diseño experimental a ambos grupos, a posteriori implementamos un control estadístico, mediante el análisis de covarianza, que permitió cuantificar y neutralizar el efecto de las diferencias iniciales en la sub-escala de comprensión. Complementariamente, como los resultados lo señalan, la covariada no resultó significativamente asociada ni al tiempo de reacción, ni tampoco a la proporción de respuestas correctas. Así entonces, nuestros análisis permiten mantener la confianza en las diferencias observadas en el experimento, que tienen que ver con modalidades de procesamiento distintas entre los niños con TEA y con DT.

\section{Referencias}

American Psychiatric Association (1994). Diagnostic and statistical manual of mental disorders (DSM-IV) (4th ed.). Washington, DC: American Psychiatric Association.

Araya, K., Araya, C., Chaigneau, S. E., Martínez, L. y Castillo, R. D. (2009). La influencia de los procesos controlados en el razonamiento con 
la Teoría de la Mente (ToM) en niños con y sin discapacidad intelectual. Revista Latinoamericana de Psicología, 41, 197-211.

Baron-Cohen, S., Leslie, A. y Frith, U. (1985). Does the autistic child have a "theory of mind"? Cognition, 21, 37-46.

Baron-Cohen, S., Leslie, A. y Frith, U. (1986). Mechanical, behavioural and intentional understanding of picture stories in autistic children. British Journal of Developmental Psychology, 4, 113-125.

Baron-Cohen, S., Ring, H., Moriarty, J., Shmitz, P., Costa, D. y Ell, P. (1994). Recognition of mental state terms: a clinical study of autism, and a functional neuroimaging study of normal adults. British Journal of Psychiatry, 165, 640-649.

Brown, H. M., Oram-Cardy, J. y Johnson, A. (2013). A meta-analysis of the reading comprehension skills of individuals on the autism spectrum, Journal of Autism and Developmental Disorders, 43, 932-955. doi 10.1007/s10803-012-1638-1.

Charman, T., Baron-Cohen, S., Swettenham, J., Baird, G., Cox, A. y Drew, A. (2000). Testing joint attention, imitation, and play as infancy precursors to language and theory of mind. Cognitive Development, $15,481-498$.

Dennis, M., Lazenby, A. y Lockyer, L. (2001). Inferential language in highfunction children with autism. Journal of Autism and Developmental Disorders, 31, 47-54.

Dennis, M., Lockyer, L., Lazenby, A., Donnelly, R., Wilkinson, M. y Schoonheyt, W. (1999). Intelligence Patterns Among Children with High-Functioning Autism, Phenylketonuria, and Childhood Head Injury. Journal of Autism and Developmental Disorders, 29, 5-17.

Harris, G., Chabris, C., Clark, J., Urban, T., Aharon, I., Steele, S., McGrath, L., Condouris, K. y Tager-Flusberg, H. (2006). Brain activation during semantic processing in autism spectrum disorders via functional magnetic resonance imaging. Brain and Cognition, 61, 54-68.

Happé, F. (1993). Communicative competence and theory of mind in autism: A test of relevance theory. Cognition, 48, 101-119.

Happé, F. (1994). Wechsler IQ profile and theory of mind in autism: A research note. Journal of Child Psychology and Psychiatry, 35, 1461-1471.

Happé, F. (1995). The role of age and verbal ability in the theory of mind task performance of subjects with autism. Child Development, 66, 843-855.

Hermosilla Valencia, M. (1982). La Escala de Inteligencia de Wechsler para Adultos (WAIS): Manual. Santiago de Chile: Pontificia Universidad Católica de Chile.

Lecannelier, F. (2004). Los aportes de la teoría de la mente (ToM) a la psicopatología del desarrollo. Terapia Psicológica, 22, 61-67.

Liebal, K., Behne, T., Carpenter, M. y Tomasello, M. (2009). Infants use shared experience to interpret pointing gestures. Developmental Science, 12, 264-271.

Loukusa, S., Leinonen, E., Kuusikko, S., Jussila, K., Mattila, M-L., Ryder, N., Ebeling, H. y Moilanen, I. (2007). Use of context in pragmatic language comprehension by children with Asperger syndrome or highfunctioning autism. Journal of Autism and Developmental Disorders, 37,1049-1059.

Moore, C., Bryant, D. y Furrow, D. (1989). Mental terms and the development of certainty. Child Development, 60, 167-171.

Onishi, K. H. y Baillargeon, R. (2005). Do 15-month-old infants understand false beliefs? Science, 308, 255-258.

Paivio, A., Yuille, J. C. y Madigan, S. A. (1968). Concreteness, imagery, and meaningfulness values for 925 nouns. Journal of experimental psychology, 76, 1-25.

Papafragou, A., Cassidy, K. y Gleitman, L. (2007). When we think about thinking: The acquisition of belief verbs. Cognition, 105, 125-165.

Ramírez, V. y Rosas, R. (2007). Estandarización del WISC-III en Chile: Descripción del Test, Estructura Factorial y Consistencia Interna de las Escalas. Psykhe, 16, 91-109.

Schopler, E., Reichler, R. J. y Renner, B. R. (1988). The Childhood Autism Rating Scale: CARS. Los Ángeles, CA: Western Psychological Sciences.

Siegel, D., Minshew, N. y Goldstein, G. (1996). Wechsler IQ profiles in diagnosis of high-functioning autism. Journal of Autism and Developmental Disorders. 26. 389-406.
Sullivan, K., Zaitchik D. y Tager-Flusberg H. (1994). Preschoolers can attribute second-order beliefs. Developmental Psychology, 30, 395-402.

Symons, D. (2004). Mental state discourse, theory of mind, and the internalization of self-other understanding. Developmental Review, 24, 159-188.

Tager-Flusberg, H. (1992). Autistic Children's Talk about Psychological States: Deficits in the Early Acquisition of a Theory of Mind. Child Development, 63, 161-172.

Tesink, C. M. J.Y., Buitellar, J. K., Petersson, K.M., van der Gaag, R.J., Teunisse, J-P. y Hagoort, P. (2011). Neural correlates of language comprehension in autism spectrum disorders: When language conflicts with world knowledge. Neuropsychologia, 49, 1095-1104. doi:10.1016/j. neuropsychologia.2011.01.018.

Tirassa, M., Bosco, F. y Colle, L. (2006). Rethinking the ontogeny of mindreading. Consciousness and Cognition, 15, 197-217.

Toichi, M. y Kamio, Y. (2002). Long-term memory and levels-of-processing in autism. Neuropsychologia, 40, 964-969.

Toichi, M. y Kamio, Y. (2003). Long-term memory in high-functioning autism: Controversy on episodic memory in autism reconsidered. Journal of Autism and Developmental Disorders, 33, 151-161.

Tomasello, M. (1996). Piagetian and Vygotskian approaches to lenguage adquisition. Human Development, 39, 269-276.

Wechsler, D. (1999). Escala de inteligencia de Wechsler para niños revisada (5 ed.). Madrid: TEA.

Ziatas, K., Durkin, K. y Pratt, C. (1998). Belief Term Development in Children with Autism, Asperger Syndrome, Specific Language Impairment, and Normal Development: Links to Theory of Mind Development. Journal of Child Psychology and Psychiatry, 39, 755-763. 